\title{
Ab-Initio Study of Electronic and Structural Properties for BaSnO3 Compound Using DFT Calculations and FP-LAPW Technique in Wein2k Software
}

\section{Zubair Ashraf ( $\nabla$ zubair@stu.pku.edu.cn ) \\ Peking University https://orcid.org/0000-0002-6909-5795 \\ Daud Rafique \\ University of the Punjab Quaid-i-Azam Campus: University of the Punjab \\ Tahir Mehmood \\ University of the Punjab Quaid-i-Azam Campus: University of the Punjab \\ Daniyal Akbar \\ University of the Punjab Quaid-i-Azam Campus: University of the Punjab}

\section{Research Article}

Keywords: PPV(Perovskites photovoltaic), SP(Structural Properties), EP (Electronic Properties) DFT (Density Functional Theory)

Posted Date: March 24th, 2021

DOI: https://doi.org/10.21203/rs.3.rs-325590/v1

License: (c) (1) This work is licensed under a Creative Commons Attribution 4.0 International License. Read Full License 
Preprint submitted to; Journal of Materials Science: Materials in Electronics

Re: Ab-initio study of electronic and Structural properties for $\mathrm{BaSnO}_{3}$

Compound using DFT calculations and FP-LAPW Technique in wein2k Software

Zubair Ashraf ${ }^{\text {n**, }}$, Mphil Physics, Ph.d Material Science

Daud Rafique ${ }^{b}$ Mphil Physics ,

Tahir Mehmood ${ }^{b}$ Mphil Physics

Danial Akbar ${ }^{[}$Mphil Physic, Ph.d

* Department of Materials Science and Engineering, Department of Energy and Resources

Engineering College of Engineering, Peking University

${ }^{\mathrm{b}}$ Department of the Physics, University of the Punjab Lahore, Pakistan.

\begin{abstract}
(Perovskites Photovoltaic) PPV cells are the hottest topics in solar cells in the recent years, because of the remarkable structural and electronic properties and hence rapid progress in material science. The Challenge associated with high-mobility $\mathrm{BaSnO}_{3}$ films is to grow. It shows high carrier mobility and UV-visible transparency has been attracting more and more attention as a very promising component for the next generation opto-electronics. Here, we demonstrate a Structural and Electronics properties ( $\mathrm{Sp}$ and $\mathrm{Ep}$ ), To characterize this compound theoretical calculation have been performed by using first principal method and the results show $\mathrm{BaSnO}_{3}$ is conductor at $0 \mathrm{eV}$ i.e. room temperature and gaining energy make more conduction transferring more electrons from conduction to valence bands. $\mathrm{BaSnO}_{3}$ shows 5.78e.v maximum for the conduction. We have studied this compound in ideal cubic phase. At $0^{\circ}$ Kelvin calculation are performed to get different properties. No experimental studies have been done on this compound. And it was difficult to accumulate its experimental data. WC-GGA is used for the study of structural properties of $\mathrm{BaSnO}_{3}$. This Correlation potential can also be used for the calculation of the various perovskite. Depending on the cubic ( $\mathrm{ABX} 3$ ) composition, perovskites exhibit a wide range of structural and electronic properties, which are optimized for different applications.
\end{abstract}

Corresponding Author: Zubair Ashraf (+923082528918)

Email: Zubair@stu.pku.edu.cn 
Keywords: PPV(Perovskites photovoltaic), SP(Structural Properties), EP (Electronic Properties) DFT (Density Functional Theory)

Corresponding Author: Zubair Ashraf (+923082528918)

Email: Zubair@stu.pku.edu.cn

\section{Contents}

1.0 Introduction 3

1.1 Stability Conditions of All Perovskites 4

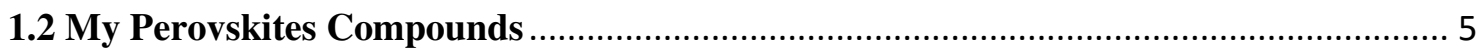

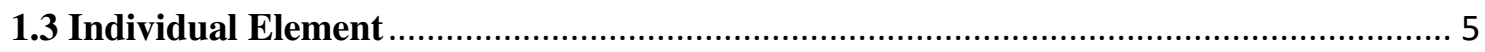

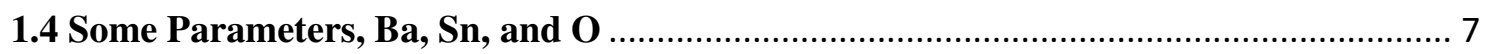

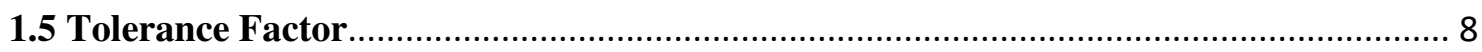

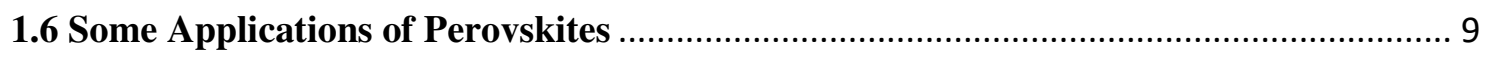

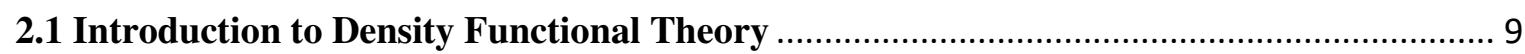

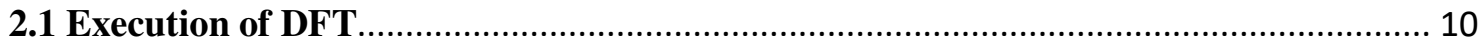

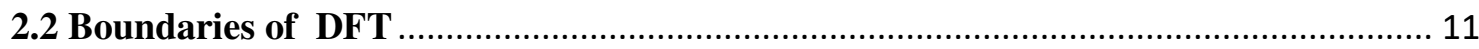

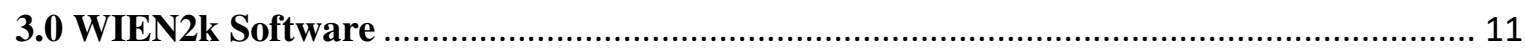

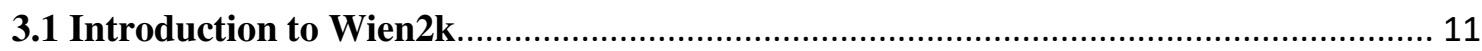

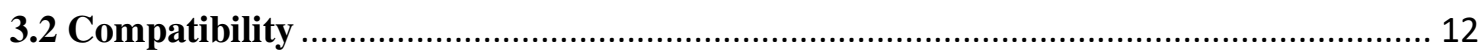

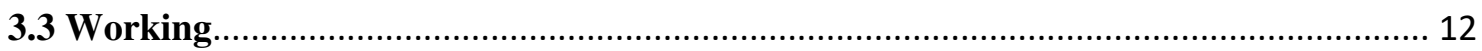

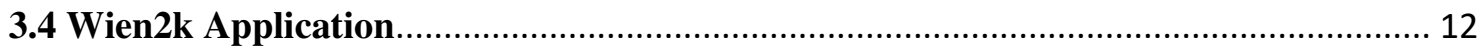

Corresponding Author: Zubair Ashraf (+923082528918)

Email: Zubair@stu.pku.edu.cn 
3.6 Structure Generation

3.7 View Structure

3.8 Initialize the Calculation.

3.9 Volume Optimization Curve 14

3.10 Evaluation of Properties 15

4. Results and Discussion 15

4.1 General Behavior of $\mathrm{BaSnO}_{3}$ 15

4.2 Structural Properties of $\mathrm{BaSnO}_{3}$ 15

4.3 Structure View of the BaSnO 17

4.4 Optimization Curve BaSnO3 17

4.5 Electronic Properties 18

5.0 References: 21

\subsection{Introduction}

High-mobility of the perovskite $\mathrm{BaSnO}_{3}$ films are of significant interest as new wide bandgap semi-conductors for the power electron as a new transparent conducting oxide and as a wide bandgap semiconductor for power electronics. To characterize this compound theoretical calculation have been performed by using first principal method and the results show $\mathrm{BaSnO} 3$ is conductor at $0 \mathrm{eV}$ i.e. Perovskites belongs to oxide family which has studied yet. They show many important and interesting most properties. Perovskites exhibit polarization if we apply electric field. The basic purposes of this thesis are "The first 


\section{Preprint submitted to; Journal of Materials Science: Materials in Electronics}

principal study of electronic properties for $\mathrm{BaSnO}_{3}$ compound using DFT calculations in wein $2 \mathrm{k}$ code". According to the best of my knowledge this work has been done for the very first time.[1]

\subsection{Stability Conditions of All Perovskites}

The conditions required for a stable perovskite is:

i. Valance bond should be balanced.

ii. Ionic radii must fulfill the condition of Goldschmidt's criteria

Goldschmidt perform great work on the study of perovskites, He determine the tolerance factor for perovskites. [ $1, \underline{2}]$
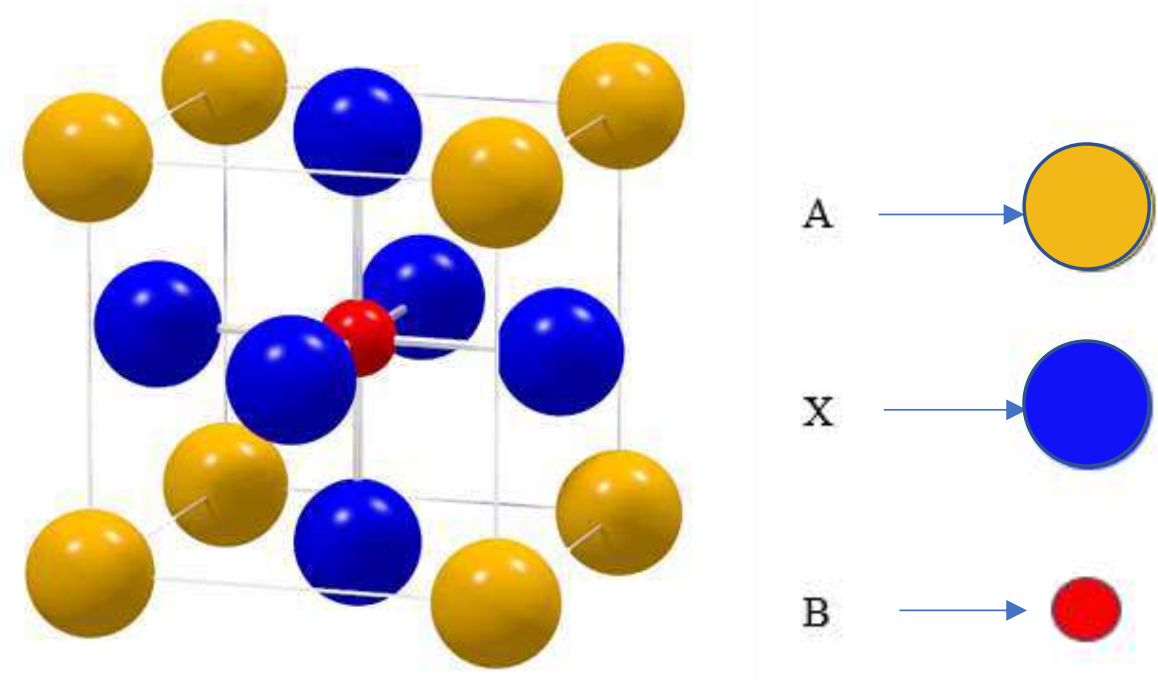

Figure (1.1): The basic cubic structure of Perovskites.

These compounds are stable in nature perovskite general structure is cubic but in actual it occurs at pseudo cubic [ [3] . at the corner there is atom A and at the center there is atom B and Atom $\mathrm{X}$ is at the face center of cube.

\section{Table (1.1): Occupation of atoms in simple perovskites.}

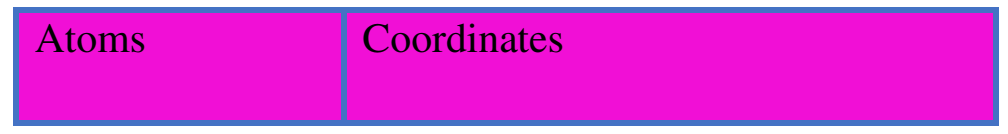




\begin{tabular}{|l|l|}
\hline A Cation & $(0,0,0)$ \\
\hline B cation & $(1 / 2,1 / 2,1 / 2)$, \\
\hline$X$ anion & $(1 / 2 ; 1 / 2,0),(1 / 2,0,1 / 2)(0,1 / 2,1 / 2$, \\
& )$[4]$ \\
\hline
\end{tabular}

\subsection{My Perovskites Compounds}

By using general formula of ABX3, My perovskites compound on which my searching is continuous, is Barium Tin Oxide or also known as Barium titanite.

\subsubsection{BaSnO3}

It is a semiconducting oxide with wide band gap of $3.1 \mathrm{eV}$. Recently, it is discovered that BaSnO3 doped with few percent of Lanthanum exhibits unusually high electrical mobility[5] , at room temperature and superior thermal stability at high temperatures [6].

It has a very low dielectric response (range from 4.9-6.6). This oxide is stable against zone center phonons in cubic perovskite structure as well as $\mathrm{KTaO} 3$ and $\mathrm{BaZro3}$ also same oxides as Barium tin oxides.

\subsection{Individual Element}

\section{$\underline{\text { 1.3.1 Barium (Ba) }}$}

It was found by S.H. Davy in England in 1808. It is named after Greek word "Barys" which means heavy. Symbolically it is represented by $B a$. It is s-block element and a member of period 6 with atomic number 56. It is fifth element of group two. It is alkaline earth metal which is very reactive. It is metallic element which exist is soft solid and it is silvery white like lead in pure form. It is good electrical conductor. [7] It easily reacts with water and alcohol. In glasses and paints small proportions of barium elements are added. Barium in salt form also used in flames to change the color to green. It has body centered cube crystal structure[7].

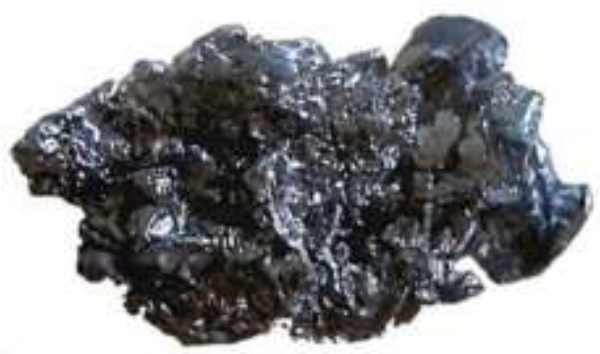

Figure (1.3.1): An Ore of Barium

Corresponding Author: Zubair Ashraf (+923082528918)

Email: Zubair@stu.pku.edu.cn 


\section{Preprint submitted to; Journal of Materials Science: Materials in Electronics}

\subsubsection{Tin (Sn)}

General symbol for the Tin is (Sn). It is not present in earth in free element form. The most famous ore for the Tin is Cassiterite that is Tin Oxide SnO2. It is anglo-Saxon word which is derived from Latin "Stannum" means Tin. It has Atomic number is 50. Electronic configuration is $(\mathrm{Kr}) 4 d^{10} 5 s^{2} 5 p^{2}$.

In ancient times conversion of other metal into gold was done by Tin which is knows as Alchemy[트, ㅇ]. Its crystal structure is existing into two forms that is cubic and tetragonal known as alpha-tin and beta-tin. It is used in marine environments because it is fungicide and bactericide. Usually it is silvery white metal with highly crystalline structure.

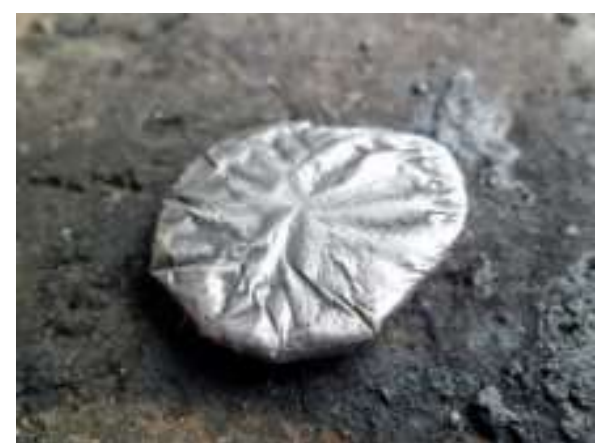

Figure (1.3.2): An ore of Tin It is p-block element in fifth period of periodic table. It is metallic element of fourth member of group 14 [9]. 


\subsubsection{Oxygen $(O)$}

Most important part of our life. It is chemical element having atomic number 8 and mass number 15.999. it is denoted by (O). It is found in non-metal group. It is oxidizing agent and second most electronegative element in periodic table[8]. It is third most plentiful gas on our planet after hydrogen. It is colorless, odorless in gaseous form, but in liquid or solid form it is blue in color. It was named by Antoin lovoiseir in 1977[요, $\underline{9}$.

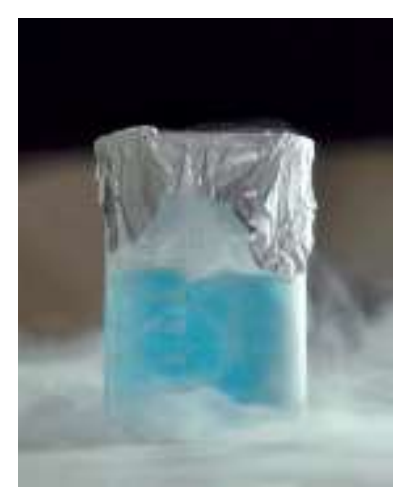

Figure (1.3.2): Oxygen in liquid form

\subsection{Some Parameters, Ba, Sn, and O}

The values of different parameters related with different assets are given as :

Table (1.2): Some important parameters of Ba, Sn, and Oxygen.

\begin{tabular}{|c|c|c|c|}
\hline Characteristic & Barium & Tin & Oxygen \\
\hline Symbol & Ba & Sn & O \\
\hline Atomic No, & 56 & 50 & 8 \\
\hline Group & 2 & 5 & 16 \\
\hline Period & 6 & 5 & 2 \\
\hline Density $(g / c c)$ & 3.51 & 7.30 & 1.42 \\
\hline Melting Point & $7^{\circ} 5^{\circ} \mathrm{C}$ & $231.97^{\circ} \mathrm{C}$ & $-219^{\circ} \mathrm{C}$ \\
\hline Boiling Point & $1897^{\circ} \mathrm{C}$ & $2602^{\circ} \mathrm{C}$ & $-183^{\circ} \mathrm{C}$ \\
\hline $\begin{array}{l}\text { Covalent Radius } \\
\text { (̊) }\end{array}$ & 1.98 & 1.41 & 66 \\
\hline
\end{tabular}

Corresponding Author: Zubair Ashraf (+923082528918)

Email: Zubair@stu.pku.edu.cn 
Preprint submitted to; Journal of Materials Science: Materials in Electronics

\begin{tabular}{|l|l|l|l|}
\hline Oxidization State & +2 & +4.2 & -2 \\
\hline Specific Heat & $0.204(\mathrm{~J} / \mathrm{gk})$ & $\mathbf{0 . 2 2 7}(\mathrm{J} / \mathrm{gk})$ & $\mathbf{0 . 1 3 0}(\mathrm{J} / \mathrm{gk})$ \\
\hline Lattice structure & $\begin{array}{l}\text { Body-center } \\
\text { cube }\end{array}$ & Tetragonal & Monoclinic at solid \\
\hline Appearance & $\begin{array}{l}\text { soft, silver- } \\
\text { white metal }\end{array}$ & $\begin{array}{l}\text { silvery-white, soft, } \\
\text { ductile-metal }\end{array}$ & $\begin{array}{l}\text { Liquid, and solid oxygen } \\
\text { Is pale blue [9, 10] }\end{array}$ \\
\hline
\end{tabular}

\subsection{Tolerance Factor}

It was determined in 1926 by Victor. M Goldschmidt. Tolerance factor was introduced to measure the distortion in perovskites from their ideal cubic structure. There is great importance of Cationic and Anionic radii of atoms in perovskite compound which plays an important role in the alteration of ideal cubic structure[11]. The deviation of orthogonal, rhombohedral can be determined by using of G. schmidt tolerance factor is given as:

$$
\mathrm{t}=r_{A}+r_{X} / \sqrt{2}\left(r_{B}+r_{X}\right)
$$

where $r_{A}$ and $r_{B}$ are the ionic radii of the cations and $r_{X}$ is the ionic radii of anion[12]. For the ideal cubic perovskites values of the tolerance factor should be equal to 1 . But in the case of the distorted perovskites that value changed from 1. Equation 1.1 describes that sum of ionic radii is nearly equal to the bond length[13]. Tolerance factor for the different structure is shown in table 1.3.

Table (1.3): The values of tolerance factor for different structure.

\begin{tabular}{|l|l|l|l|}
\hline Tolerance Factor & Structure & Ionic Radii & Example \\
\hline$>1$ & Hexagonal & $\begin{array}{l}\text { A and B both are so } \\
\text { small }\end{array}$ & BaNiO3 \\
\hline
\end{tabular}


Preprint submitted to; Journal of Materials Science: Materials in Electronics

\begin{tabular}{|l|l|l|l|}
\hline 0.9 to 1 & Cubic & $\begin{array}{l}\text { A and B have ideal } \\
\text { size }\end{array}$ & SrTiO3 \\
\hline 0.71 to 0.9 & Orthorhombic & A is so small than B & CaTiO3 \\
\hline$<0.71$ & $\begin{array}{l}\text { Trigonal } \\
\text { Different structure }\end{array}$ & $\begin{array}{l}\text { A and B both have } \\
\text { similar Radii }\end{array}$ & FeTiO3[14, 15] \\
\hline
\end{tabular}

\subsection{Some Applications of Perovskites}

Due to some remarkable physical, chemical and structural properties, they have an important place in the world of science research. These multi purposes causes many applications of the perovskites and thus have an important place in the Industry field. A perovskites oxide Barium titrate have so much variety of applications because of ionic and mixed conductivity it is used as capacitors, thermistors, temperature co-efficient, and piezoelectric devices (in which device produces AC voltage when we apply mechanical stress) etc [13]. perovskites that are used in highly effective photovoltaic are synthetic perovskites[15]. They are prepared same as solar cell by method of thin film[14]. They are also used to produced Laser light, when LiAlO3 is doped with neoydium they produce laser light of 1080nm. Those perovskites have high resistivity are used in dielectric material, some of them are LaTiO3, LaVO3, etc. and some of them are good conductor because B atom in $\mathrm{ABO} 3$ having oxidization state of $+2[\underline{16}]$. They are also used in oxygen sensor like SrTiO3.

\subsection{Introduction to Density Functional Theory}

To understand the internal forces of the atoms when they join in the solids and to study the different properties (like thermal, electronic, magnetic and optical, etc.) of the solids, different theories were postulated with the passage of time. Nowadays the most acceptable theory in regard with the measurement of the different properties of matter or solids is "Density Functional Theory" . It was developed by W. Kohn and J. Pople. Walter Kohn was awarded by the Noble Prize in 1998 for the development of DFT.

This theory was developed by the combination of the two theorems which was given in the 1960's [1, 18]by: 
Hohenberg-Kohn in 1964.

Kohn-Sham in 1965.

A simple glance on the background in table is given as:

Table (2.1): Development of DFT from beginning.

\begin{tabular}{|l|l|}
\hline Year & Work \\
\hline $1920 s$ & Introduction of Thomas fermi model. \\
\hline $1964 s$ & Hohenberg-Kohn proves existence of DFT. \\
\hline $1965 s$ & Kohn-Sham scheme introduction. \\
\hline $1970 s$ to early & LDA, DFT becomes useful. \\
\hline $1985 s$ & Incorporation of DFT into molecular dynamics. \\
\hline $1998 s$ & $\begin{array}{l}\text { Noble prize awarded to Walter Kohn in Chemistry for } \\
\text { work in DFT. [18, } 19]\end{array}$ \\
\hline
\end{tabular}

\subsection{Execution of DFT}

In an external potential of atomic nuclei Density Functional Theory is the most successful theory of today science and used for the study of the properties of system of electrons. The major advantage of the Density Functional Theory is reduction 3N degree of freedom of a system to three degree of freedom that made Density Functional Theory able to solve complicated system[20]. It gives ground state properties of material which further used to understand the behavior of that material with other materials, in general it gives the theoretical approach to the physical properties as well as electronic, optical, thermal, magnetic and electrostatic properties on the ground state level[21]. In respect to the Chemistry approach Density Functional Theory is also a successful theory, in which it has many applications. 
It is used to measure the following bonds:

Bond length.

$>$ Bond angles.

$>$ Nature of Bonds[22]. It has importance in the biological field, material like nanotubes, proteins, and carbon. Optimization as well as atomization energies are also measurable because of Density Functional Theory. Density Functional Theory has direct relation of ground state energy of the system with its electron density. This electron density is actually main variable on which whole Density Functional Theory exists[21, 22].

\subsection{Boundaries of DFT}

DFT cannot be useful to determine each and every properties and has deficiencies in determining some properties listed below:

\section{Band Gap}

Absolute values of band gap cannot be determine.

Superconductivity

Accurate prediction is not available in DFT.

High temperature properties.

Unable to calculate the properties at higher temperature close to melting points[19, $\underline{21}, \underline{22}]$.

\subsection{WIEN2k Software}

\subsection{Introduction to Wien2k}

To calculate electronic, structural, magnetic, thermal, optical and many others properties of the materials in SSP in which we use the concepts of density functional theory these all can be measured in single package called Wien2k software. One of the most and accurate methods of full potential is linearized augmented plane wave method (LAPW), which is used to calculate band structures and it is the base of the wien $2 \mathrm{k}$ package. Wien $2 \mathrm{k}$ deals with the electrons and their relativistic effects and many others features like quantum mechanical properties.

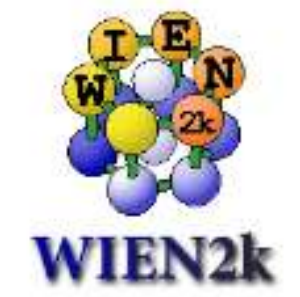

Fig 3.1

Corresponding Author: Zubair Ashraf (+923082528918)

Email: Zubair@stu.pku.edu.cn 


\section{Preprint submitted to; Journal of Materials Science: Materials in Electronics}

The first copyright version was Wien, it is introduced by Peter Blaha, George Madsen, K.H

Schwarz, and D. Kvasnicka[1]. 2000+ groups have the license which shows the importance. Many precise and updated version of the UNIX have been developed which were Wien93, Wien95, and Wien97. Today the most updated version of the Wien2k is Wien2k_18.2[1].

\subsection{Compatibility}

Wien $2 \mathrm{k}$ is a computer program having FORTRAN90 language, which require UNIX operating system, and the installation in which program are connected through C-Shell script.

Following are the computers systems on which Wien2k can be run successfully.

- IBM RS6000

- Intel Pentium PC, s working Under LINUX

- HP

- SUN

- DEC Alpha

- SGI

Hardware requirement are different for the different cases for example having 10 atoms per unit cell run on the Pentium-II PC with memory $128 \mathrm{MB}$ under LINUX. But for the powerful PC disk space up too few GB is recommended.

But the demand for the software is 1 GB disk space and $512 \mathrm{MB}$ cache memory.

\subsection{Working}

We are dealing our compounds in ideal conditions in material science and in ideal crystals. We consider the crystal at $0^{\circ} \mathrm{k}$ temperature, thus we can calculate many properties at ground state level. Like every software we use exchange correlation potential in Wien2k also [ $\underline{1}$, $\underline{23}, \underline{24}]$.

\subsection{Wien2k Application}

To understand the structure and many properties of an atom or compound it has many applications in the field of Science.

$\checkmark$ It is used to solve Kohn-Sham Equation of DFT.

$\checkmark$ For the calculation of structural properties. 
$\checkmark$ It is computational program which is used to study the ideal compound.

$\checkmark$ It is used to calculate electronic properties, density of the states, and band structure.

$\checkmark$ Optical, elastic and magnetic properties are measured through Wien2k software.

$\checkmark$ It is used for the calculation of the electron energy loss spectrum, core loss spectrum [25].

It determines the elastic constants for cubic cases.

\subsection{Software codes}

It consists of many independent FORTRAN90 programs, which are linked to each other's through C-shell script. This software can be executed using any web browser or w2web interface. We used wien2k software by using short commands. The main steps for the software are;

- Define structure.

- Initialize all commands.

- SCF (run self-consistent-field

These are the basic building block for the run to this software[26].

\subsection{Structure Generation}

First, we need to generate structure of the compound to find out the properties of the compound. We need to click on the struct-gen command and then put all the values of the compound like position of each atom, number of atoms, lattice constant, angels of atoms, and space group. By entering all commands, we have to click on Save structure, after that RMT is automatically set, by continuing editing structure file is saved[3] $]$.

\subsection{View Structure}

After input all data of the given compound and executing then click on the View_struct to view the structure of the compound.

\subsection{Initialize the Calculation}

After saving the structure, now we are going to initialize the calculations for properties with symmetry. Init_lapw term is used to initialize the command, initialization depends upon the 6 properties, these properties perform different task. 
These are given as:

* X-nn

In this term 'nn' stands for nearest neighbor distance, this is used to calculate the distance between spheres and for checking the overlapping of all atomic spheres.

* X-sgroup

It specifies the space group and point group of the non-equivalent atoms. It can also distinguish the different atoms by using value of the $\mathrm{Z}$ uniquely. It can find out the smallest unit cell and also shift the origin of a unit cell.

* X-symmetry

Space group symmetry is checked by the 'X-symmetry' command. This program gives the atomic densities of the compound.

* X-lstart

It is also called LSDA programing it is start by Hartree units at input and end on Rydberg units at output. This program can also produce atomic potential.

* X-kgen

This produce $\mathrm{k}$-mesh by converging all the $\mathrm{k}$ points in an irreducible region of the brillouin zone.

* X-dstart

To make initial density of crystalline solids calculation from $\mathrm{x}$-lstart is superimposed. This program is called “'superposition of the Atomic Density”.'[27]

\subsection{Volume Optimization Curve}

To find out shape and size of the unit cell having total energy optimization process is used. Shape and size are very important parameters. We use experimental values as a input, to calculate the total energy of the perovskites compound this can be measured by the use of Murnaghan Equation.

$$
\mathbf{E}(\mathbf{v})=\mathbf{E}_{\mathrm{o}}(\mathbf{v})+\mathbf{B V} / \dot{\mathbf{B}}(\dot{\mathbf{B}}-\mathbf{1})\left[\mathbf{B}\left\{\mathbf{1}-\mathrm{V}_{\mathrm{o}} / \mathbf{V}\right\}+\left(\mathbf{v}_{\mathrm{o}} / \mathbf{v}\right) \dot{\mathbf{B}}-\mathbf{1}\right.
$$

In equation (3.1) we have:

$\mathrm{E}_{\mathrm{o}}(\mathrm{v})=$ Ground state energy

$\mathrm{V}=$ ground state volume.

$\mathrm{B}=$ bulk modulus.

$\mathrm{B}^{\prime}=$ bulk modulus pressure.

Corresponding Author: Zubair Ashraf (+923082528918)

Email: Zubair@stu.pku.edu.cn 


\section{Preprint submitted to; Journal of Materials Science: Materials in Electronics}

$\mathrm{E}(\mathrm{v})$ energy verses volume Curve.

(3.1) equation is the second order equation which gives the ground state energy and volume. Thus, we can calculate energy by entering the values of volume[28]. Therefore, its graph is always parabola along $\mathrm{y}$-axis. The graph is plotted between $\mathrm{V}$ and $\mathrm{E}$. This process is known as Volume optimization, the lattice constant is also determined from the equilibrium volume. The range of the graph tells us how much time is required to give volume optimization[29].

\subsection{Evaluation of Properties}

After SCF cycle, the properties of the given compound is obtained and calculated, like, electronic, optical, etc. can be obtained using wien2k software. since, we know the properties of the material or compound, we can use these into the field of science[30].

\section{Results and Discussion}

\subsection{General Behavior of $\mathrm{BaSnO}_{3}$}

In order to characterize this compound theoretical calculation have been performed by using first principle method. I have studied this compound in ideal cubic phase. At $0^{\circ}$ Kelvin calculation are performed in order to get different properties. No experimental studies have been done on this compound. And it was difficult to accumulate its experimental data.

$\mathrm{BaSnO}_{3}$ having space group 221_pm3m. Barium Stannate $\left(\mathrm{BaSnO}_{3}\right)$ depending on temperature it have different types of structures. It shows many dramatic properties for example ionic conductivity, superconductivity, colossal magnetoresistance[30-32], as well as ferromagnetism and ferroelectricity and semi-conductivity70. It also shows piezoelectric and optical properties which have great importance in microelectronics and

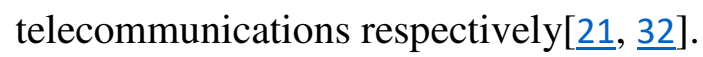

In this chapter I will study structural and electrical properties of $\mathrm{BaSnO}_{3}$ along with graphs in WEIN2k software.

\subsection{Structural Properties of $\mathrm{BaSnO}_{3}$.}

WC-GGA is used for the study of structural properties of both $\mathrm{BaSnO}_{3}$. This Correlation potential can also be used for the calculation of the various perovskites. It is comparatively 


\section{Preprint submitted to; Journal of Materials Science: Materials in Electronics}

most accurate technique having less uncertainty. For the very first compound the orientation is adjusted in such a way that $\mathrm{Ba}$ is placed at origin and location of Atom in compound is $(0,0,0)$, and for $\mathrm{Sn}$ it is in the center of the cubic structure with location $(1 / 2,1 / 2,1 / 2)$, and for the last atom oxygen is in the face centered having location $(1 / 2,1 / 2,0),(1 / 2,0,1 / 2)$, and $(0,1 / 2,1 / 2)$. For the structural properties of $\mathrm{BaSnO}_{3}$ it needs input parameters like, lattice constant, space group, and atomic positions of the every atom etc. These parameters are given as below in table.

\section{Table 4.1 Input Parameters for Structural Properties}

\begin{tabular}{|c|c|c|}
\hline \multicolumn{3}{|l|}{$\mathrm{BaSnO}_{3}$} \\
\hline Parameters & Names & Values \\
\hline Angles & $\begin{array}{l}\mathbf{A} \\
\boldsymbol{\beta} \\
\gamma\end{array}$ & $\begin{array}{l}90^{\circ} \\
90^{\circ} \\
90^{\circ}\end{array}$ \\
\hline $\begin{array}{l}\text { Atomic Number } \\
\text { (Z) }\end{array}$ & $\begin{array}{l}\text { Ba } \\
\text { Sn } \\
\text { O }\end{array}$ & $\begin{array}{l}56 \\
50 \\
8\end{array}$ \\
\hline Position & $\begin{array}{l}\text { Ba } \\
\text { Sn } \\
\text { O }\end{array}$ & $\begin{array}{l}(0,0,0), \quad(1 / 2,1 / 2,1 / 2), \\
\{(1 / 2,1 / 2,0),(1 / 2,0,1 / 2), \\
(0,1 / 2,1 / 2)\}\end{array}$ \\
\hline Lattice Constant & $\begin{array}{l}\text { A } \\
\text { B } \\
\text { C }\end{array}$ & $\begin{array}{l}4.189 \AA \\
4.189 \AA \\
4.189 \AA\end{array}$ \\
\hline Space Group & 221 & Pm-3m \\
\hline Potential & $\begin{array}{l}\text { Exchange } \\
\text { correlation }\end{array}$ & WC-GGA \\
\hline RMT & $\begin{array}{l}\text { Ba } \\
\text { Sn } \\
\text { O }\end{array}$ & $\begin{array}{l}2.50 \AA \\
1.97 \AA \\
1.70 \AA\end{array}$ \\
\hline
\end{tabular}




\subsection{Structure View of the BaSnO}

I have performed all steps as mentioned above, for the calculation of the structural properties required. Steps are done one by one to calculates the structural properties. By using WEIN2k package structure of the $\mathrm{BaSnO}_{3}$ can be obtained which is given in below figure (4.1). In this structure ' $\mathrm{Ba}$ ' is at

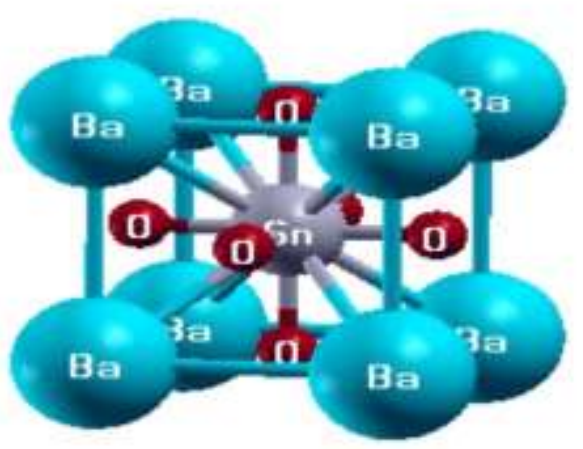
the corner of the cube and ' $\mathrm{Sn}$ ' is in the center of the cube, and ' $\mathrm{O}$ ' is at the face centered position. Figure 4.1 ideal cubic unit cell of $\mathrm{BaSnO}_{3}$

\subsection{Optimization Curve BaSnO3}

In case of $\mathrm{BaSnO}_{3}$, the increase in volume of the unit the values of the energy decreases of the crystal structure. The certain value of the volume which is minimum energy of the volume, after this value energy of the volume increases, this is called optimized equilibrium energy, because the system is in the equilibrium.

The Energy-Volume curve for this compound is plotted in given figure, The value of the GSE and $\mathrm{V}$ for $\mathrm{BaSnO}_{3}$ is obtained 29088.815 Ry and 494 (a.u) ${ }^{3}$ respectively. Some other important parameters of the $\mathrm{BaSnO}_{3}$ can also be obtained like,

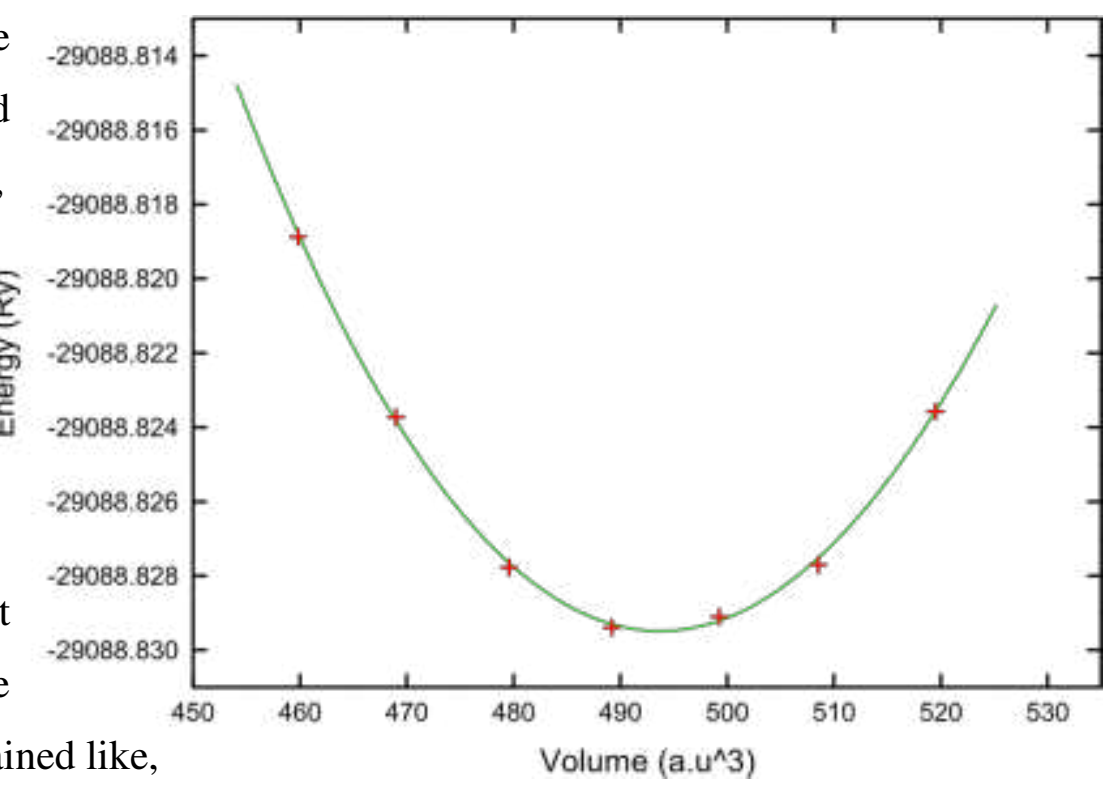

Figure 4.3 The total energies of the cell volume of $\mathrm{BaSnO}_{3}$ pressure derivatives of the bulk modulus and Bulk modulus at 0 value[21, $\underline{22}, \underline{33}]$. Bulk Modulus is the ability to stand against compression with its original unit volume, and its 


\section{Preprint submitted to; Journal of Materials Science: Materials in Electronics}

shape is also retainable, up to the value of pressure which is applied. It can also be obtained as:

$\mathrm{B}=\frac{\text { Stres }}{\text { Strain }}$

All these properties are calculated by $\mathrm{Wu}$ Cohen-GGA Approximation using Wien2k package.

\subsection{Electronic Properties}

Those properties which are related to the electrons are called electronic properties, which gives the behavior of the electrons within the crystal. Some of the useful result that I have determined about electrons are:

Density of the States

Band Gap

Electrons Density

Each of them is explained as:

\subsubsection{Density of states}

It can be defined as "Energy levels available to be occupied by electrons per unit energy range". Mathematically it is given as:

$$
\mathrm{D}(\mathrm{E})=\frac{d \Omega(E)}{d E}
$$

Whereas $d \Omega$ represents the no. of the states, these states can be written as:

$\mathrm{D}(\mathrm{E})=\sum \int\left(\mathrm{d}^{3} \mathrm{k} \times \delta\left(\mathrm{E}-\varepsilon_{\mathrm{n}}(\mathrm{k})\right) /(2 \pi)^{3}\right.$

$\varepsilon_{\mathrm{n}}$ is eigen value energy[34] I have drawn the graph of the density of the states, with the help of Wien2k software,

This graph are plotted between energy and DOS.

The unit used for the energy is $\mathrm{eV}$ and for the

$\mathrm{DoS}$ is (states/eV), the energy is taken along the $\mathrm{x}$-axis and DoS is on the $\mathrm{y}$-axis. This graph show increase and decrease in the energy and DOS.

Five atoms combine to make the unit cell of each of my compound $\left(\mathrm{BaSnO}_{3}\right)$. Each atom shows

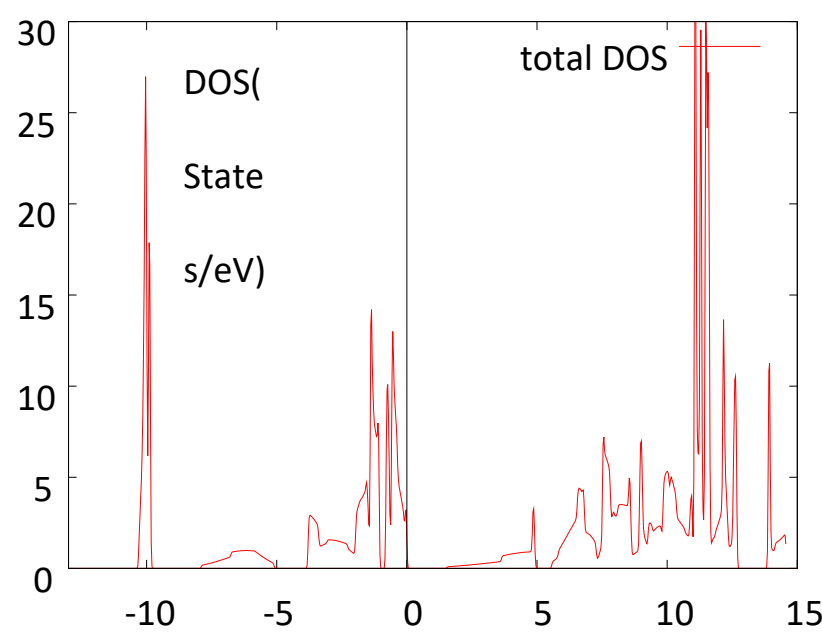




\section{Preprint submitted to; Journal of Materials Science: Materials in Electronics}

different configuration and valence electrons as well as conduction states, every peak Figure 4.5 Total density of states for $\mathrm{BaSnO}_{3}$

In the Graph shows different orbitals[28]. Valence electrons are nearly very close to the conduction's electrons, when they jump from one band to another attain some energy as photons, and hence peaks show only for the valence electrons in the DOS.

\subsubsection{Electrons Density}

The measure of the probability of the electrons around the atom is called electron density, which gives the bonding between atoms[32]. ED has relation with wavefunction of the electrons. Electron density gives the information about bond nature and about interaction between atoms.

For finding electron density of my compounds I have used 2-D plots for the electron density,

The electrons density for the perovskite's compounds $\mathrm{BaSnO}_{3}$ is given as;

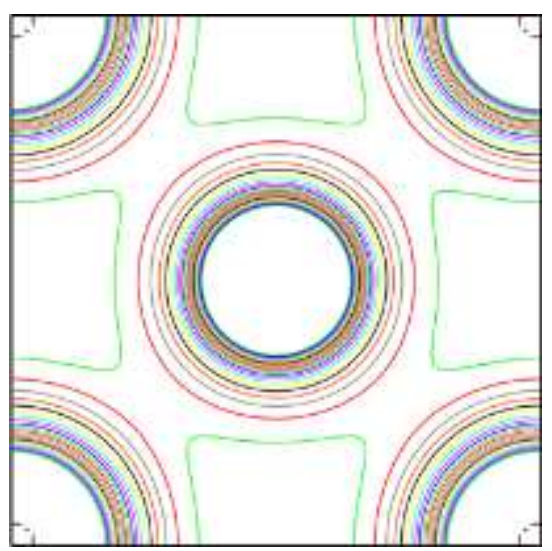

Figure 4.5 (a) Electron Density for $\mathrm{BaSnO}_{3}$

The very first graph is for the $\mathrm{BaSnO}_{3}$ in which symmetric is shown for the electrons density when magnetic field is absence, the main center portion shows combine effect of $\mathrm{Ba}-\mathrm{Sn}$, the light green color is for the oxygen representation, the upper corner shows Ba-O interactions and $\mathrm{Sn}-\mathrm{O}$ shows lower corner interaction [21, 31, 32]. 


\section{Preprint submitted to; Journal of Materials Science: Materials in Electronics}

\subsubsection{Band Gap}

It can be defined as:

The energy of the states in which the electrons are found or exits are called Energy bands or Allowed Gap. On the other hand, those energy states in which the electrons cannot be exists are called Forbidden Gaps or Electronic Band Gaps [28]. The graph for the band structure for the $\mathrm{BaSnO}_{3}$ is also studies by use of the Wien2k package are given as:

This graph is highly symmetric with the symmetric points of the Brillion Zone. With presence of the Electric field[31, 32], the different color and the curves shows the path of Electrons in Motions. Each curve shows the independent path of the electrons. The value of the energy is plotted against $y$-axis and, those of negative value

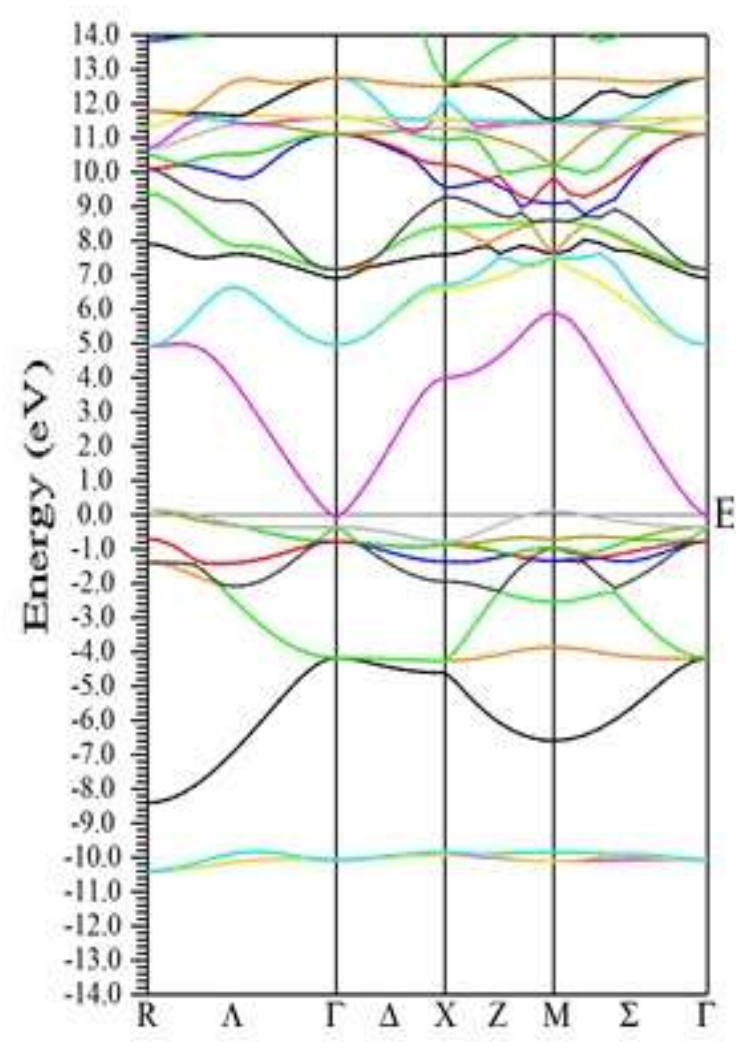

Figure 4.6 Band Gap for $\mathrm{BaSnO}_{3}$ shows the energy below the fermi level which are valence and lower bands $[\underline{21}, \underline{22}, \underline{33}]$. Those bands above the fermi levels are positive values showing conduction bands. The graph is shown for the $\mathrm{BaSnO}_{3}$ which seems to same band structure throughout the structure thus showing $\mathrm{BaSnO}_{3}$ is a unique compound. In the graph the symmetry points are plotted along $\mathrm{x}$-axis. In $\mathrm{BaSnO}_{3}$ the maximum points of the valence band are shown in grey color lies in $\mathrm{M}$ symmetry point whereas the minimum points are lies in $\Gamma$ symmetry points, are purple in color which seems $\mathrm{BaSnO}_{3}$ is categories as indirect Band Gap Compound. It is also named as M-Г Indirect Band Gap Compound [17, 34-38].

Wide bandgap perovskite oxides with high room temperature conductivities and structural compatibility with a diverse family of organic/inorganic perovskite materials are of significant interest as transparent conductors and as active components in power electronics. Such materials must also possess high room temperature mobility to minimize power consumption and to enable high-frequency applications.

\section{Acknowledgements:}

Corresponding Author: Zubair Ashraf (+923082528918)

Email: Zubair@stu.pku.edu.cn 


\section{Preprint submitted to; Journal of Materials Science: Materials in Electronics}

The authors are thankful to the Lab facilities provided by University of the Punjab. There is no conflict of intertest between authors.

\subsection{References:}

1. Blaha, P., et al., WIEN2k: An APW+lo program for calculating the properties of solids. $\mathbf{J}$ Chem Phys, 2020. 152(7): p. 074101.

2. Athawale, A.A., M.S. Bapat, and P.A. Desai, Hydrothermal preparation of BaSnO3 and Au-BaSnO3 nanorods. J Nanosci Nanotechnol, 2008. 8(8): p. 4258-62.

3. Prakash, A., et al., Wide bandgap BaSnO3 films with room temperature conductivity exceeding 10(4) S cm(-1). Nat Commun, 2017. 8: p. 15167.

4. Arezoomandan, S., et al., THz characterization and demonstration of visibletransparent/terahertz-functional electromagnetic structures in ultra-conductive La-doped BaSnO3 Films. Sci Rep, 2018. 8(1): p. 3577. 


\section{Preprint submitted to; Journal of Materials Science: Materials in Electronics}

5. Buannic, L., et al., Probing cation and vacancy ordering in the dry and hydrated yttriumsubstituted BaSnO3 perovskite by NMR spectroscopy and first principles calculations: implications for proton mobility. J Am Chem Soc, 2012. 134(35): p. 14483-98.

6. Donval, G., et al., A hybrid method using the widely-used WIEN2k and VASP codes to calculate the complete set of XAS/EELS edges in a hundred-atoms system. Phys Chem Chem Phys, 2017. 19(2): p. 1320-1327.

7. Eustace, D.A., D.W. McComb, and A.J. Craven, Probing magnetic order in EELS of chromite spinels using both multiple scattering (FEFF8.2) and DFT (WIEN2k). Micron, 2010. 41(6): p. 547-53.

8. Hebert, C., Practical aspects of running the WIEN2k code for electron spectroscopy. Micron, 2007. 38(1): p. 12-28.

9. Galazka, Z., et al., Melt growth and properties of bulk BaSnO3 single crystals. J Phys Condens Matter, 2017. 29(7): p. 075701.

10. Hebert, C., J. Luitz, and P. Schattschneider, Improvement of energy loss near edge structure calculation using Wien2k. Micron, 2003. 34(3-5): p. 219-25.

11. Holec, D., et al., Electron energy loss near edge structure (ELNES) spectra of AlN and AlGaN: a theoretical study using the Wien $2 k$ and Telnes programs. Micron, 2008. 39(6): p. 690-7.

12. Huang, C., et al., A facile peroxo-precursor synthesis method and structure evolution of large specific surface area mesoporous BaSnO3. Inorg Chem, 2015. 54(8): p. 4002-10.

13. Kim, D.W., et al., BaSnO3 perovskite nanoparticles for high efficiency dye-sensitized solar cells. ChemSusChem, 2013. 6(3): p. 449-54.

14. Kim, M., et al., Oxygen-Vacancy-Introduced BaSnO3-delta Photoanodes with Tunable Band Structures for Efficient Solar-Driven Water Splitting. Adv Mater, 2019. 31(33): p. e1903316.

15. Kim, Y.M., et al., Interface polarization model for a 2-dimensional electron gas at the BaSnO3/LaInO3 interface. Sci Rep, 2019. 9(1): p. 16202.

16. Lee, J.H., et al., Transparent $p$-CuI/n-BaSnO3-delta heterojunctions with a high rectification ratio. J Phys Condens Matter, 2017. 29(38): p. 384004.

17. Zhu, L., et al., Mesoporous BaSnO3 layer based perovskite solar cells. Chem Commun (Camb), 2016. 52(5): p. 970-3.

18. Allam, O., et al., Density Functional Theory - Machine Learning Approach to Analyze the Bandgap of Elemental Halide Perovskites and Ruddlesden-Popper Phases. Chemphyschem, 2018. 19(19): p. 2559-2565. 


\section{Preprint submitted to; Journal of Materials Science: Materials in Electronics}

19. Azam, S., et al., Revealing the optoelectronic properties of Re-based double perovskites using the Tran-Blaha modified Becke-Johnson with density functional theory. J Mol Model, 2020. 26(6): p. 158.

20. English, N.J., Dynamical properties of organo lead-halide perovskites and their interfaces to titania: insights from Density Functional Theory. Heliyon, 2020. 6(3): p. e03427.

21. Xiao, Z., et al., Correction to "Intrinsic Instability of Cs2In(I)M(III)X6 $(M=B i, S b ; X=$ Halogen) Double Perovskites: A Combined Density Functional Theory and Experimental Study". J Am Chem Soc, 2017. 139(27): p. 9409.

22. Xiao, Z., et al., Intrinsic Instability of $C s 2 \operatorname{In}(I) M(I I I) X 6(M=B i, S b ; X=$ Halogen $)$ Double Perovskites: A Combined Density Functional Theory and Experimental Study. J Am Chem Soc, 2017. 139(17): p. 6054-6057.

23. Mizoguchi, H., et al., Strong near-infrared luminescence in BaSnO3. J Am Chem Soc, 2004. 126(31): p. 9796-800.

24. Nahm, H.H., et al., Amorphous Mixture of Two Indium-Free BaSnO3 and ZnSnO3 for ThinFilm Transistors with Balanced Performance and Stability. ACS Appl Mater Interfaces, 2020. 12(3): p. 3719-3726.

25. Ovcharov, A.V., et al., Microstructure and superconducting properties of high-rate PLDderived GdBa2Cu3O7-delta coated conductors with $\mathrm{BaSnO} 3$ and $\mathrm{BaZrO3}$ pinning centers. Sci Rep, 2019. 9(1): p. 15235.

26. Prakash, A., et al., Separating Electrons and Donors in BaSnO3 via Band Engineering. Nano Lett, 2019. 19(12): p. 8920-8927.

27. Rajamanickam, N., et al., Efficiency enhancement of cubic perovskite BaSnO3 nanostructures based dye sensitized solar cells. Phys Chem Chem Phys, 2016. 18(12): p. 8468-78.

28. Sanchela, A.V., et al., Thermopower Modulation Clarification of the Operating Mechanism in Wide Bandgap BaSnO3 -SrSnO3 Solid-Solution Based Thin Film Transistors. Small, 2019. 15(8): p. e1805394.

29. Shin, S.S., et al., Colloidally prepared La-doped BaSnO3 electrodes for efficient, photostable perovskite solar cells. Science, 2017. 356(6334): p. 167-171.

30. Wang, W.Y., et al., Atomic mapping of Ruddlesden-Popper faults in transparent conducting BaSnO3-based thin films. Sci Rep, 2015. 5: p. 16097.

31. Wang, Y., et al., High-mobility two-dimensional electron gas in SrGeO3-and BaSnO3based perovskite oxide heterostructures: an ab initio study. Phys Chem Chem Phys, 2016. 18(46): p. 31924-31929. 


\section{Preprint submitted to; Journal of Materials Science: Materials in Electronics}

32. Wu, M., et al., The Role of Annealing Process in Ag-Based BaSnO3 Multilayer Thin Films. Nanoscale Res Lett, 2016. 11(1): p. 369.

33. Yue, J., et al., Depletion Mode MOSFET Using La-Doped BaSnO3 as a Channel Material. ACS Appl Mater Interfaces, 2018. 10(25): p. 21061-21065.

34. Yun, H., et al., Microstructure characterization of $\mathrm{BaSnO} 3$ thin films on LaAlO3 and PrScO3 substrates from transmission electron microscopy. Sci Rep, 2018. 8(1): p. 10245.

35. Yun, H., et al., STEM beam channeling in BaSnO3/LaAlO3 perovskite bilayers and visualization of 2D misfit dislocation network. Ultramicroscopy, 2020. 208: p. 112863.

36. Yun, H., et al., Metallic line defect in wide-bandgap transparent perovskite BaSnO3. Sci Adv, 2021. 7(3).

37. Zhang, Y., M.P. Sahoo, and J. Wang, Tuning the band gap and polarization of BaSnO3/SrSnO3 superlattices for photovoltaic applications. Phys Chem Chem Phys, 2017. 19(10): p. 7032-7039.

38. Zhang, Y., et al., Strain-induced ferroelectricity and lattice coupling in BaSnO3 and SrSnO3. Phys Chem Chem Phys, 2017. 19(38): p. 26047-26055. 
Figures
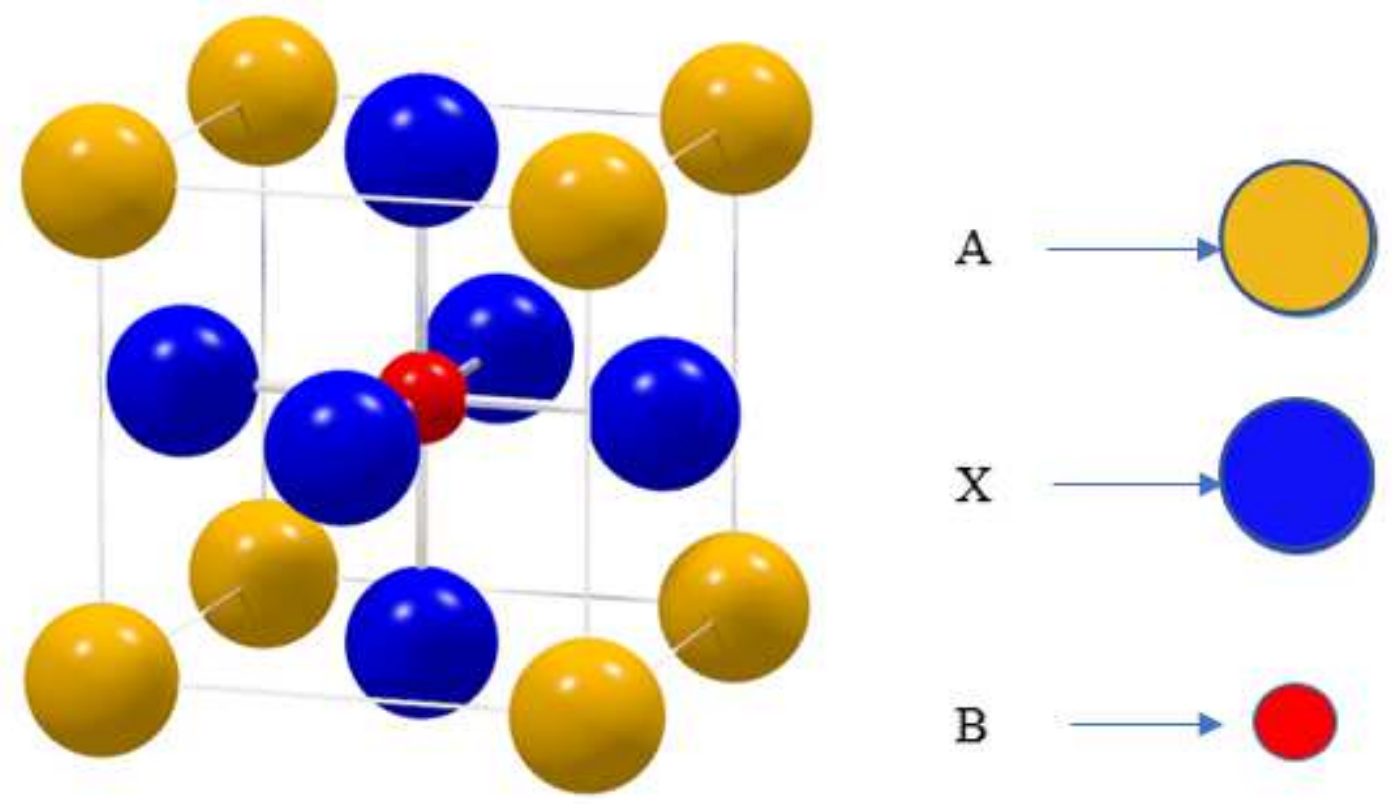

Figure 1

The basic cubic structure of Perovskites.

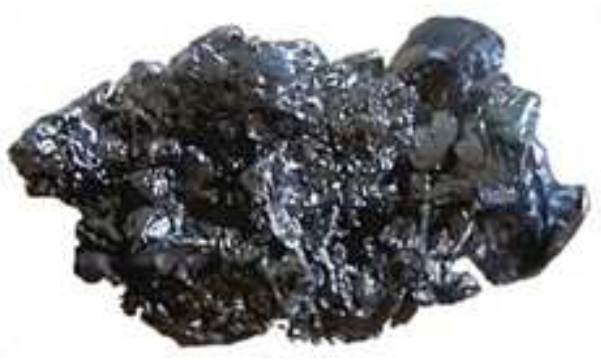

Figure 2

An Ore of Barium

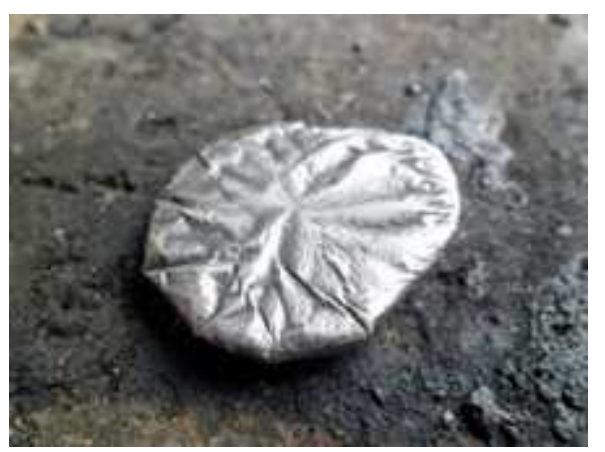

Figure 3

An ore of Tin 


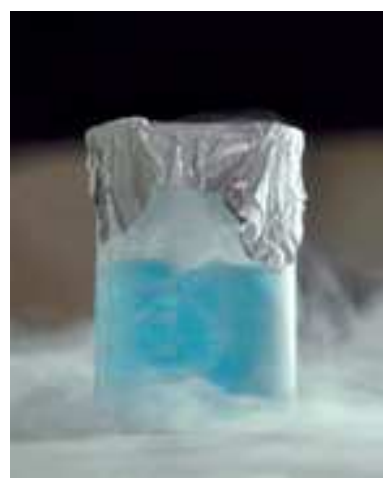

\section{Figure 4}

Oxygen in liquid form

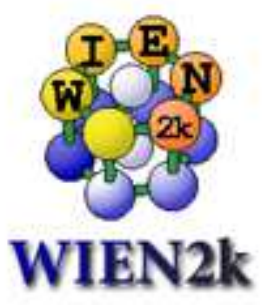

Figure 5

Introduction to Wien2k

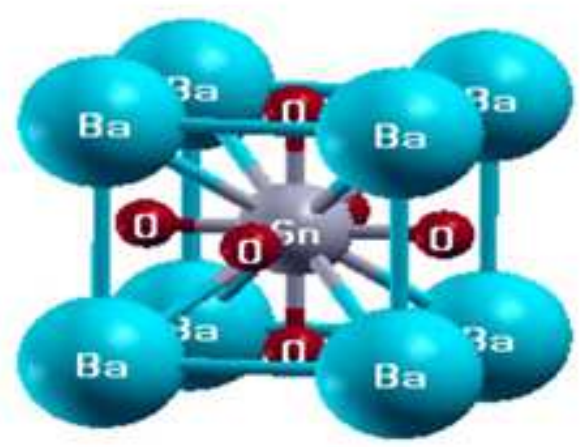

Figure 6

ideal cubic unit cell of $\mathrm{BaSnO}$ 


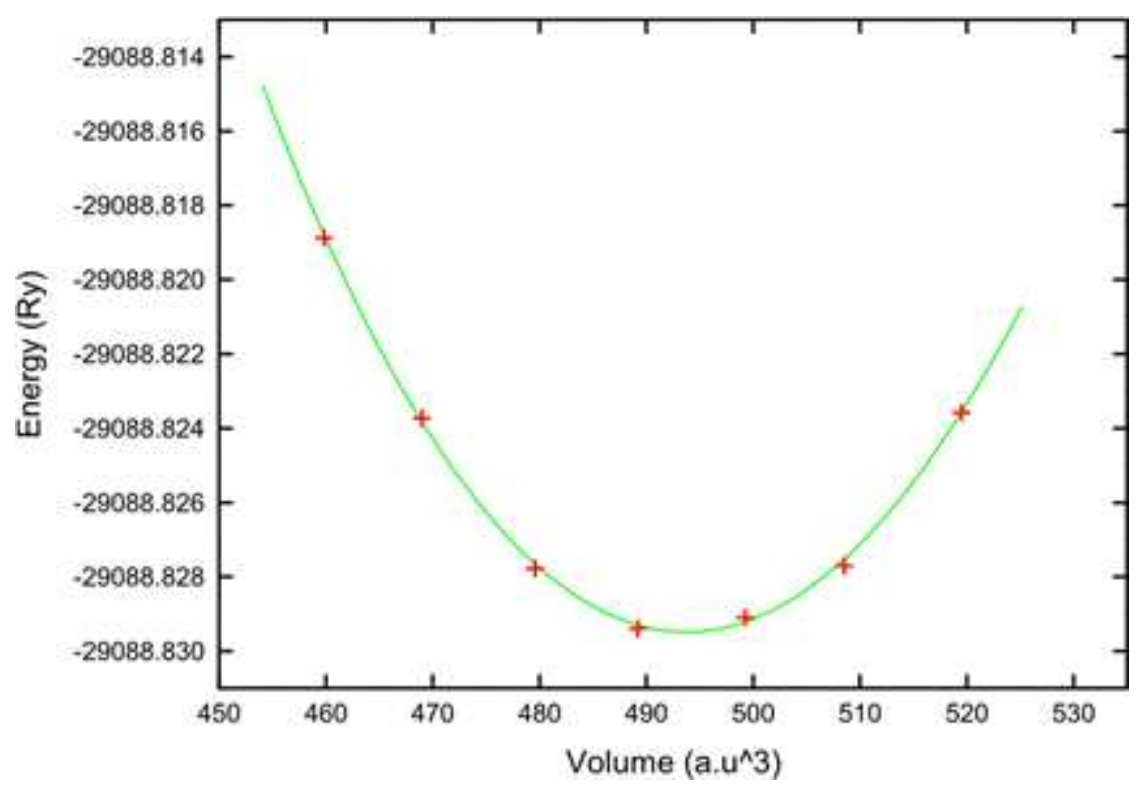

Figure 7

The total energies of the cell volume of $\mathrm{BaSnO} 3$

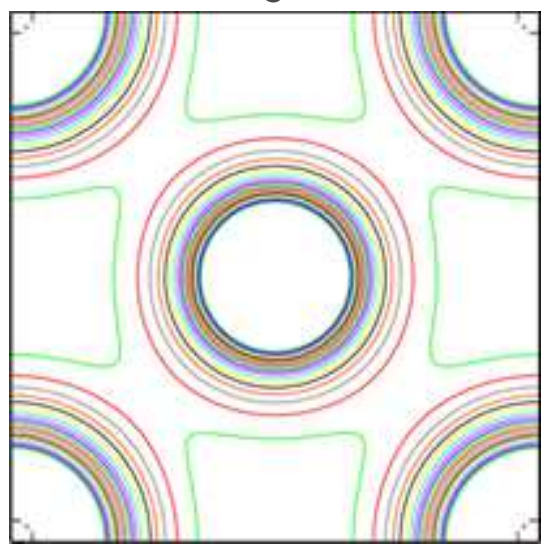

Figure 8

(a) Electron Density for $\mathrm{BaSnO} 3$ 


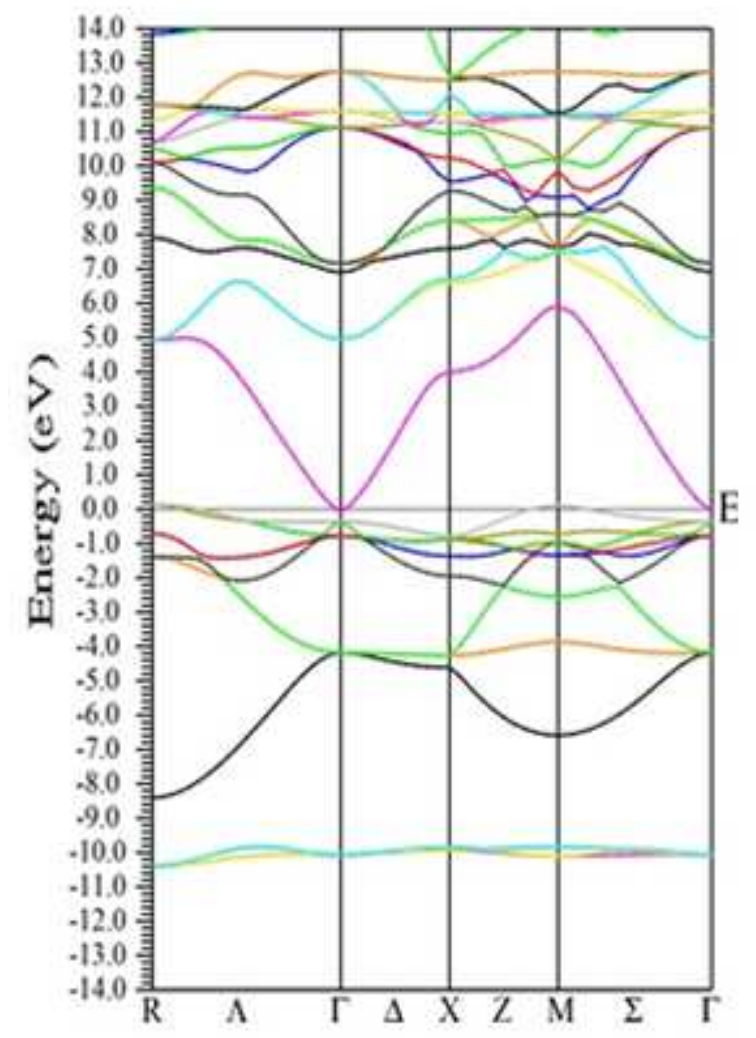

Figure 9

Band Gap for BaSnO3

\section{Supplementary Files}

This is a list of supplementary files associated with this preprint. Click to download.

- GraphicalAbstractnew1.docx 